\title{
Comparison of Oncoplastic Breast Surgery, Non- oncoplastic Breast Conserving Surgery and Mastectomy in Early Breast Cancer: A Single Center Retrospective Study
} \author{
Orhan Alimoglu, MD ${ }^{1}$ \\ 'Istanbul Medeniyet University Medical Faculty, Department of General Surgery, Istanbul, Turkey \\ ${ }^{2}$ Istanbul Kadikoy Florence Nightingale Hospital, Department of Pathology, Istanbul, Turkey \\ ${ }^{3}$ Istanbul Medeniyet University Medical Faculty, Department of Radiology, Istanbul, Turkey \\ 4Istanbul GOP Hospital, Department of Medical Oncology, Istanbul, Turkey
}

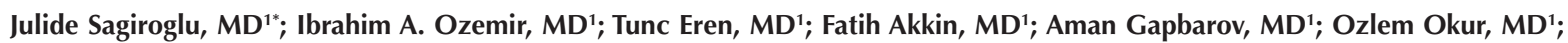
Hakan Baysal, MD'; Ozgur Ekinci, MD'; Ayse B. Ceyran, MD²; Esra Tozan Bayrak, MD³; Turkan Ozturk, MD'; Gurhan Bas, MD;

\begin{abstract}
Background: Surgical procedures applied in the treatment of early breast cancer (EBC) to achieve satisfactory oncological results lie in a wide spectrum. There has been a major shift toward less-invasive treatments during the past decades. We compared the outcomes of oncoplastic breast surgery (OBS), non-oncoplastic breast conserving surgery (NBCS) and mastectomy in the treatment of $\mathrm{EBC}$.

Methods: The records of 412 patients with EBC who underwent OBS, NBCS or mastectomy at our institution between January 2012 and June 2019 were retrospectively analyzed. Postoperative complications, local recurrences (LR) and disease-free survival (DFS) were compared between the groups. EBC patients with unilateral stage-I, Ila and IIb tumors were studied. All patients received adjuvant, targeted and/or endocrine therapy according to the tumor characteristics, followed by radiotherapy (all OBS and NBCS cases, and selected mastectomy patients).

Results: Postoperative complications were similar in all groups except for six fat necrosis and partial nipple-areola necrosis in two diabetic patients treated with OBS. Re-excision rate was lower in OBS (6.5\%) than NBCS (8\%). There was no statistical difference between the groups regarding recurrence $(P=1.000)$ or DFS $(P=0.937)$.

Conclusion: OBS, NBCS and mastectomy are equally acceptable procedures in EBC in terms of both oncological and surgical aspects.

Keywords: Breast conserving, Early breast cancer, Mastectomy, Oncoplastic surgery, Survival

Cite this article as: Sagiroglu J, Ozemir la, Eren T, Akkin F, Gapbarov A, Okur O, et al. Comparison of Oncoplastic Breast Surgery, Non-oncoplastic Breast Conserving Surgery and Mastectomy in Early Breast Cancer: A Single Center Retrospective Study. Arch Iran Med. Arch Iran Med. 2021;24(5):390-396. doi: 10.34172/aim.2021.56
\end{abstract}

Received: July 28, 2020, Accepted: February 27, 2021, ePublished: May 1, 2021

\section{Introduction}

Early breast cancer (EBC) is defined as disease confined to the breast with or without regional lymph node involvement, and the absence of distant metastatic disease. Surgical procedures in the management of EBC have progressed towards less aggressive techniques in order to achieve better aesthetic outcomes with oncologic safety. ${ }^{1-3}$ Mastectomy is defined as resection of the entire breast with the tumor, where as in non-oncoplastic breast conserving surgery (NBCS), the breast tumor is resected with safe oncological margin from the breast without applying reconstructive techniques to reshape the remaining breast tissue. Oncoplastic breast surgery (OBS) enables excision of the tumor with safe margins while accomplishing better physical integrity of the breast by application of reconstructive techniques. ${ }^{4} \mathrm{~A}$ benefit of OBS is maintaining the breast with the best possible aesthetic results and good control of tumor margins, while facilitating resection of larger tumors and offering patients the privilege of breast conservation. ${ }^{5-9}$

Multidisciplinary preoperative evaluation would be mandatory to obtain the best results in all types of breast surgery. This multidisciplinary team includes breast oncologic surgeons, breast radiologists, breast oncologists, and breast pathologists. The breast oncologic surgeon determines the optimal surgical technique for the patient, and follows with deciding the most appropriate reconstructive technique if OBS is the method of choice. Delicate preoperative planning as well as profound experience is mandatory for application of OBS in order to decrease complication rates and obtain successful results. When the surgical method of choice is NBCS or mastectomy, there will be no need for a detailed reconstructive plan. ${ }^{10-12}$ 
A pile of evidence suggested oncoplastic technique algorithms based on tumor location. Berry et al described ten oncoplastic techniques. ${ }^{13}$ Iwuchukwu et al divided the breast into seven zones, each of which corresponded with suggested mammoplasty techniques. ${ }^{14}$ The reconstruction technique is selected according to tumor location, size, and the patient's preference.

While aesthetic outcomes after OBS compared to non-oncoplastic procedures such as lumpectomy and mastectomy can be considered more satisfactory for the EBC patient, comparing the oncological safety obtained with OBS and non-oncoplastic surgical methods may be conflicting. ${ }^{7-9}$ Local tissue rearrangement in OBS may influence local recurrences (LR) and detection. Rietjens et al found that LR rates were low over long-term follow-up, with a $3 \%$ rate at 5 years and no recurrences were seen in tumors smaller than $2 \mathrm{~cm} .{ }^{15}$

The aim of this study was to compare the effects of mastectomy, NBCS and OBS in terms of complications, LR and survival in patients with EBC. We present our results in these three groups in the management of EBC.

\section{Patients and Methods}

Hospital records of patients (984 women) who had been operated for BC between January 2012 and June 2019 were analyzed, and 412 women who had unilateral EBC were included in the study. The inclusion criteria for this study were women $\geq 18$ year of age who had breast surgery for unilateral stage-I, IIa or IIb BC. The exclusion criteria were bilateral $\mathrm{BC}$, advanced $\mathrm{BC}$ beyond stage $\mathrm{IIb}$, previous radiotherapy to the breast or chest region, male gender, and being younger than 18 years of age. The included patients were retrospectively categorized in three groups of mastectomy $(n=124)$, NBCS $(n=150)$ and OBS $(\mathrm{n}=138)$. All patients received adjuvant chemotherapy (CT), targeted therapy, and/or endocrine therapy based on the tumor characteristics, followed by radiotherapy (RT) (all OBS, NBCS patients and selected mastectomy patients) on the operated breast and/or ipsilateral axilla.

\section{Preoperative Assessment and Surgery}

Diagnoses and staging were made based on preoperative physical examination, imaging (mammography, ultrasonography, magnetic resonance imaging), and biopsies (core needle and stereotactic). Treatment plans were discussed with the oncology council. Suitable patients underwent adjuvant therapy after surgery. All operations were carried out by the breast surgery team, consisting of experienced breast surgeons. Each patient was preoperatively evaluated for tumor-to-breast ratio, breast size, and skin quality. Mastectomy (simple or modified radical) was defined as complete removal of the breast from the underlying pectoralis major muscle, and patients undergoing mastectomy were selected based upon multicentricity of the tumor and the patients' own preference for this procedure. Patients with high tumor-to-breast size ratio who were unable to receive
RT (connective tissue diseases, pregnancy), and those who preferred more extensive surgery instead of having RT, also underwent mastectomy with or without axillary dissection (AD).

Resection of the breast quadrant or lump which involved the tumor, without reshaping techniques was considered as NBCS. The criteria for selection for NBCS (and OBS) included the size, depth, and location of the tumor in addition to the histologic features of the cancer. Contraindications for NBCS and OBS were multicentricity of the tumor (with diffuse micro-calcifications), especially in ductal carcinoma in situ (DCIS), mutations in the BRCA1-2 genes, connective tissue disease and pregnancy in the first trimester. Oncoplastic procedures were categorized as level I (excision of less than $20 \%$ of breast volume with reshaping mammoplasty), and level II (excision of $20 \%-50 \%$ of breast volume with reshaping mammoplasty). Patients were selected for OBS based on high tumor-to-breast volume ratios in small sized breasts, more challenging locations such as central, superomedial locations, or multifocality, rendering breast-conserving therapy difficult.

\section{Surgical Techniques}

Simple mastectomy (complete breast tissue removal with the tumor and the breast skin including the NAC without AD) or modified radical mastectomy (simple mastectomy with $\mathrm{AD}$ ) were performed in the mastectomy group. Patients in the NBCS group underwent resection of the tumor from the breast with marking the tumor bed by four clips (for boost RT preparation), and simple surgical closure of the wound. No further reconstructive procedure was considered for this group.

The optimal oncoplastic method for each patient in the OBS group was determined according to the tumor location. Preferred level II procedures were $\mathrm{V}$ scar mammoplasty $(n=8)$ for lower inner quadrant, batwing mammoplasty $(n=6)$ for upper inner quadrant, inferior pedicle/round block mammoplasty $(n=16)$ for upper pole, superior pedicle /inverted $\mathrm{T}$ or vertical scar $(n=14)$ for lower pole, racquet incision $(n=4)$ for upper outer pole, and inverted $\mathrm{T}$ with nipple-areola complex (NAC) resection $(n=6)$ for central sub-areolar tumors. Immediate expander placement $(n=6)$ was considered for patients who underwent subcutaneous mastectomy (skinsparing mastectomy with preservation of the NAC) for $\mathrm{T}_{1} \mathrm{~N}_{0} \mathrm{M}_{0}$ invasive ductal carcinoma (IDC) with widespread DCIS. ${ }^{2}$ In all OBS procedures except subcutaneous mastectomies, tumor bed clipping was performed for boost RT. All patients underwent axillary staging (via AD or sentinel lymph node biopsy [SLNB] only) according to institutional protocols.

Intraoperative frozen section was routinely applied for the OBS and NBCS groups in order to define surgical margins free of tumor. 'No ink on tumor' was accepted as satisfactory clear surgical margin free of tumor. When margin positivity was reported by the pathologist, re- 
excision specimens were verified by new frozen sections during the operation. All patients with clinically negative (non-palpable) axilla underwent SLNB using methylene blue dye. Radio-labelled sulphur colloid was used for axilla staging instead of methylene blue dye in one pregnant patient in the third trimester who underwent mastectomy. Level I-II AD was performed for patients with metastatic sentinel LNs confirmed during the operation, and for patients with clinically positive axillary LNs. Patients who underwent $\mathrm{AD}$ as well as the number of metastatic axillary $\mathrm{LN}(\mathrm{s})$ for each patient was recorded. Clinical stage, histopathological type, tumor size, grade, nodal status of axilla, estrogen receptor (ER), progesterone receptor (PR), Ki67, E-cadherin, Her2, multicentricity, and multifocality were noted.

\section{Adjuvant Therapy}

According to tumor characteristics, the patients started receiving CT within 4 weeks of surgery (postpartum for the pregnant patient). Following the completion of CT, all patients in the OBS and NBCS groups received supplementary RT at a 50-Gy dose to the entire breast and additional 10-Gy boost dose to the tumor bed, as well as to node positive axilla. In the mastectomy group, only patients with either tumors in close proximity to the pectoralis major fascia, or node positive axilla received RT (the pregnant patient received postpartum RT because of the close proximity of the tumor to the pectoralis major fascia). Adjuvant CT, endocrine therapy, and RT were administered in accordance with the clinical protocol. Her2-positive patients were given trastuzumab after the operation. After the adjuvant treatments, 5-year tamoxifen or an aromatase inhibitor regimen was started for ERpositive patients.

\section{Follow-up}

Follow-up visits were every 3 months during the first postoperative year, every 6 months in the second year, and once per year thereafter. Median follow-up was 74 months ( $r=7-89$ months). Mammography and breast ultrasonography were performed once a year. Further radiographic scanning was performed only on suspicion. New malignancy formation in the operated quadrant of the breast with the same pathological features was recorded as $L R$.

\section{Statistical Analysis}

Statistical analysis was performed using the NCSS (Number Cruncher Statistical System) 2007 Statistical Software (Kaysville, Utah, USA). Data evaluation was completed by definitive statistical methods (mean, standard deviation, median, frequency, rate, minimum and maximum). Quantitative data comparison of normally distributed variable groups ( $\mathrm{n} \geq 3$ ) was performed using the one-way ANOVA test, and confounding variables were detected by the Games-Howell test. In non-normally distributed groups ( $\mathrm{n} \geq 3$ ), the Kruskal-Wallis test was used for comparison, and the confounding variables were detected by the Mann-Whitney U test. Conformity of the quantitative data to a normal distribution was tested using the Kolmogorov-Smirnov, Shapiro-Wilk test, and graphical assessments. We used the Pearson chisquare, Fisher-Freeman-Halton, Fisher's exact and Yates' continuity correction tests (Yates' corrected chi-square) for comparison of qualitative data.

The Kaplan Meier survival analysis was used for diseasefree survival (DFS). Probability values of less than 0.01 and 0.05 were considered significant.

\section{Results}

Mean age was 53.93, SD13.77 years $(r=27-91)$. Level I OBS was performed for 78 patients, and level II for 60 patients. Median tumor diameter was $2.21 \mathrm{~cm}(\mathrm{r}=0.3-5$ $\mathrm{cm})$. The mastectomy group demonstrated greater tumor size than both the NBCS and OBS groups $(P=0.002$ and $P=0.003)$, while no difference was found between NBCS and OBS $(P=0.867)$ (Table 1$)$.

The groups were significantly different with respect to clinical stages; stage-I disease was greater in OBS than in mastectomy $(P=0.026)$, while the difference between groups in stage-II was very close to significance $(P=0.055)$. The groups revealed no difference regarding histological grade, Her2, Ki-67, E-cadherin, ER and PR expression (Table 1).

IDC was the most common tumor type in all groups and there was no difference between the groups with respect to the IDC rates $(P=0.070)$ (Table 2$)$. The mastectomy group revealed a higher invasive lobular carcinoma (ILC) rate than the OBS group $(P=0.012)$, while there was no difference when compared with the NBCS group $(P=0.187)$. When the NBCS and OBS groups were compared, the ILC rates were not different $(P=0.287)$ (Table 2).

The groups revealed a significant difference with respect to $\mathrm{AD}(P=0.003)$. The mastectomy group had a significantly higher $\mathrm{AD}$ rate than the NBCS group $(P=0.003)$, while revealing no difference compared to the OBS group $(P=0.556)$. When the OBS and NBCS groups were compared, the OBS group demonstrated a significantly higher $\mathrm{AD}$ rate than the NBCS group $(P=0.015)$.

Intraoperative frozen section necessitated 9 re-excisions in OBS (6.5\%) and 12 re-excisions in NBCS (8\%) patients for margin positivity. Re-excision specimens were verified by new frozen sections intraoperatively.

Postoperative complications such as wound dehiscence and extensive seroma formation were rare. Fat necrosis was slightly higher in OBS (4.35\%) than the other groups (M: $0.81 \%$, NBCS: $1.33 \%$ ) but the difference was not significant. Two patients with type II diabetes mellitus developed partial areola necrosis following OBS (1.45\%), which were treated by topical Hirudoid gauze application (Table 3).

The groups did not reveal a statistical difference with 
Table 1. Comparison of Clinicopathological Parameters

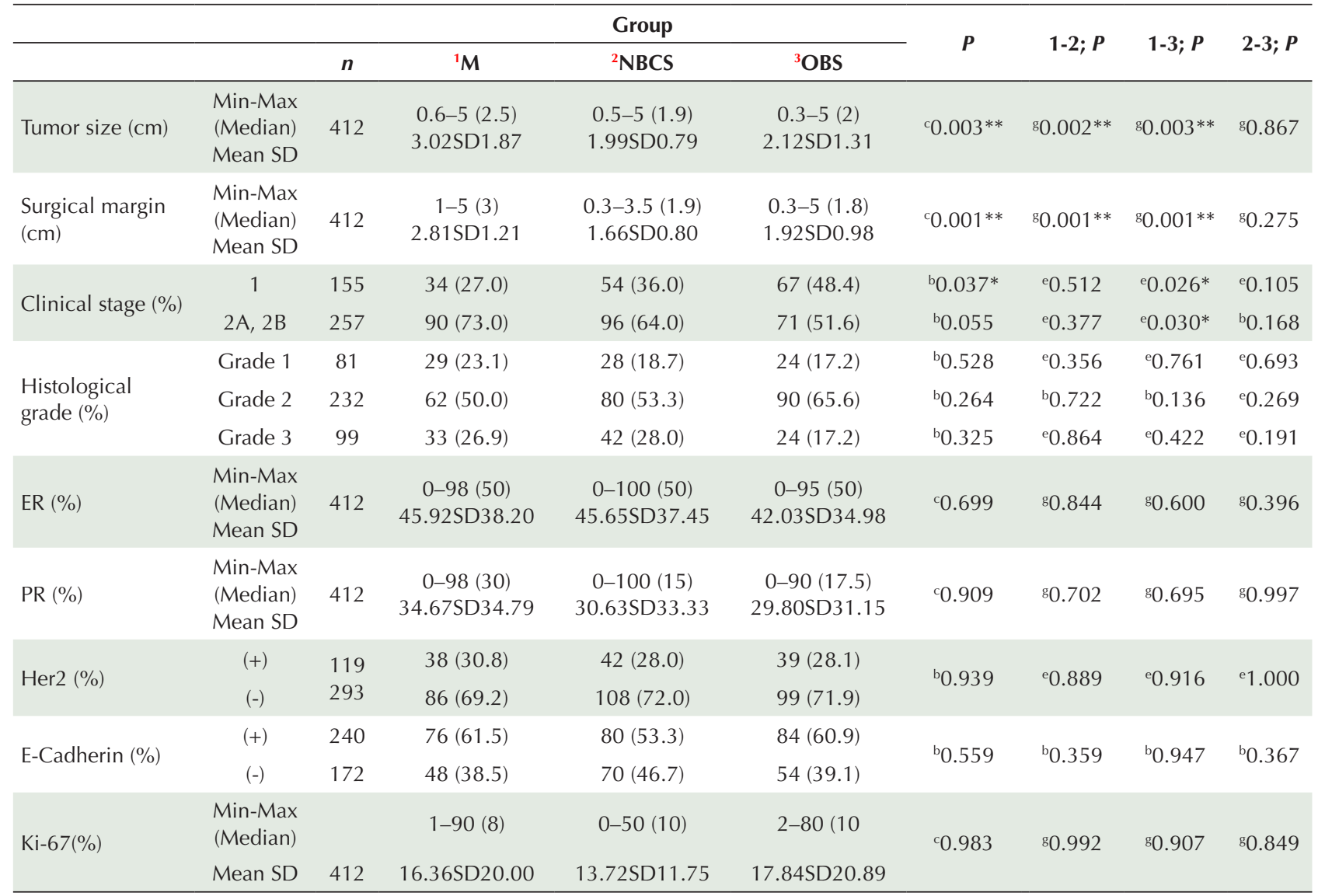

SD, Standard deviation; M, Mastectomy; NBCS, Non-oncoplastic breast conserving surgery; OBS; Oncoplastic breast surgery; ER, Estrogen receptor; PR, Progesterone receptor.

${ }^{\mathrm{a}}$ Fisher-Freeman-Halton test; ${ }^{\mathrm{b}}$ Pearson chi-square; ${ }^{\mathrm{c}}$ Kruskal-Wallis test, ${ }^{\mathrm{e}}$ Yates continuity correction test, ${ }^{\mathrm{f}}$ Fisher exact test, ${ }^{\mathrm{g}}$ Mann-Whitney test; ${ }^{*} P<0.05 ;{ }^{* *} P<0.01$.

Table 2. Comparison of Tumor Types (IDC, ILC, DCIS, LCIS, other)

\begin{tabular}{|c|c|c|c|c|c|c|c|c|c|}
\hline & & \multicolumn{4}{|c|}{ Group } & \multirow{2}{*}{$P$} & \multirow{2}{*}{$1-2 ; P$} & \multirow{2}{*}{$1-3 ; P$} & \multirow{2}{*}{$2-3 ; P$} \\
\hline & & $n$ & ${ }^{1} \mathrm{M}$ & ${ }^{2} \mathrm{NBCS}$ & ${ }^{3} \mathrm{OBS}$ & & & & \\
\hline \multirow{2}{*}{ IDC (\%) } & $(+)$ & 313 & $86(69.2)$ & $108(72.0)$ & 119 (85.9) & \multirow{2}{*}{ b0.070 } & \multirow{2}{*}{${ }^{\mathrm{e}} 0.889$} & \multirow{2}{*}{${ }^{\mathrm{e}} 0.051$} & \multirow{2}{*}{${ }^{\mathrm{e}} 0.074$} \\
\hline & $(-)$ & 99 & 38 (30.8) & 42 (28.0) & $19(14.1)$ & & & & \\
\hline \multirow{2}{*}{ ILC (\%) } & $(+)$ & 37 & $21(17.3)$ & $12(8.0)$ & $4(3.1)$ & \multirow{2}{*}{${ }^{\mathrm{a}} 0.033^{*}$} & \multirow{2}{*}{${ }^{\mathrm{e}} 0.187$} & \multirow{2}{*}{${ }^{\mathrm{f}} 0.012 *$} & \multirow{2}{*}{${ }^{f} 0.287$} \\
\hline & $(-)$ & 375 & $103(82.7)$ & $138(92.0)$ & $134(96.9)$ & & & & \\
\hline \multirow{2}{*}{ DCIS (\%) } & $(+)$ & 183 & 43 (34.6) & $80(53.3)$ & $60(43.8)$ & \multirow{2}{*}{ b0.114 } & \multirow{2}{*}{${ }^{\mathrm{e}} 0.057$} & \multirow{2}{*}{${ }^{\mathrm{e}} 0.418$} & \multirow[t]{2}{*}{${ }^{\mathrm{b}} 0.308$} \\
\hline & $(-)$ & 229 & 81 (65.4) & $70(46.7)$ & $78(56.3)$ & & & & \\
\hline \multirow{2}{*}{ LCIS (\%) } & $(+)$ & 12 & $2(1.9)$ & $8(5.3)$ & $2(1.6)$ & \multirow{2}{*}{${ }^{\mathrm{a}} 0.510$} & \multirow{2}{*}{ f0.648 } & \multirow{2}{*}{${ }^{\mathrm{f}} 1.000$} & \multirow{2}{*}{${ }^{f} 0.374$} \\
\hline & $(-)$ & 400 & $122(98.1)$ & $142(94.7)$ & $136(98.4)$ & & & & \\
\hline \multirow{2}{*}{ Other (\%) } & $(+)$ & 60 & $21(17.3)$ & $24(16.0)$ & 15 (10.9) & \multirow{2}{*}{${ }^{\mathrm{b}} 0.614$} & \multirow{2}{*}{$\mathrm{e} 1.000$} & \multirow{2}{*}{${ }^{\mathrm{e}} 0.472$} & \multirow{2}{*}{${ }^{\mathrm{e}} 0.536$} \\
\hline & $(-)$ & 352 & $103(82.7)$ & $126(84.0)$ & $123(89.1)$ & & & & \\
\hline
\end{tabular}

M, Mastectomy; NBCS, Non-oncoplastic breast conserving surgery; OBS, Oncoplastic breast surgery; IDC, Invasive ductal carcinoma; ILC, Invasive lobular carcinoma; DCIS, Ductal carcinoma in situ; LCIS, Lobular carcinoma in situ.

${ }^{\text {a }}$ Fisher-Freeman-Halton test; ${ }^{\mathrm{b}}$ Pearson chi-square; ${ }^{\mathrm{e}}$ Yates continuity correction test; ${ }^{\mathrm{f}}$ Fisher exact test; ${ }^{*} P<0.05 ;{ }^{* *} P<0.01$.

respect to multicentricity or multifocality of the tumor $(P=0.378)$. Adjuvant $\mathrm{CT}$ administration rates revealed a significant difference between the groups $(P=0.008)$. Rate of RT application was significantly lower in the mastectomy group than the NBCS and OBS groups $(P=0.001)$. Endocrine treatment rates were similar
$(P=0.153)$. Tumor recurrence was reported at one patient per group, and there was no significant difference between the groups with respect to rate and time of recurrence $(P=1.000, P=0.963)$ (Table 4).

Patients with LR demonstrated features such as 48 years of age (premenopausal), $\mathrm{T}_{3} \mathrm{~N}_{0} \mathrm{M}_{0}$, no $\mathrm{AD}, \mathrm{ER}(+), \mathrm{PR}(+)$, 
Table 3. Postoperative Complications

\begin{tabular}{lccc}
\hline & \multicolumn{3}{c}{ Group } \\
\cline { 2 - 4 } & ${ }^{1} \mathbf{M}(\boldsymbol{n})$ & ${ }^{2}$ NBCS $(\boldsymbol{n})$ & ${ }^{3}$ OBS $(\boldsymbol{n})$ \\
\hline Wound dehiscence (\%) & $2(1.61)$ & $0(0)$ & $2(1.45)$ \\
Seroma (\%) & $11(8.87)$ & $3(2)$ & $4(2.89)$ \\
Fat necrosis (\%) & $1(0.81)$ & $2(1.33)$ & $6(4.35)$ \\
NAC partial necrosis (\%) & $0(0)$ & $0(0)$ & $2(1.45)$ \\
\hline
\end{tabular}

$\bar{M}$, Mastectomy; NBCS, Non-oncoplastic breast conserving surgery; OBS, Oncoplastic breast surgery; NAC, Nipple-areola complex.

Her2(+), Ki67: 65\% in the mastectomy group (LR detected within the dermis of the surgery wound); 59 years of age (postmenopausal), $\mathrm{T}_{2} \mathrm{~N}_{1} \mathrm{M}_{0}$, positive $\mathrm{AD}$, triple negative, Ki67: 35\% in the NBCS group; 39 years of age (premenopausal), $\mathrm{T}_{2} \mathrm{~N}_{1} \mathrm{M}_{0}$, positive $\mathrm{AD}$, triple negative, Ki67: 45\% in the OBS group. Recurring tumor type was IDC in all groups.

Cumulative DFS in the $52^{\text {nd }}$ postoperative month (in which the last recurrence was recorded) was $99.3 \%$. The log rank test demonstrated no statistical difference between the groups with respect to DFS $(P=0.937)$ (Table 5). There was no exitus.

\section{Discussion}

Oncoplastic surgery is an increasingly applied procedure in BC management because of better cosmetic outcomes, higher patient satisfaction and satisfying oncologic results. ${ }^{16,17}$

Losken et al reported the LR rate for OBS and NBCS at $3.6 \%-4.7 \%$, and $7 \%$, respectively. ${ }^{18}$ According to Caruso et al, the LR rate in $\mathrm{BC}$ patients with bilateral reduction mammoplasty was $1.5 \%{ }^{19}$ In a prospective cohort study on patients with locally advanced BC undergoing OBS,
Bogusevicius et al reported an LR rate of $10 \%$ at 86 months. ${ }^{20}$ However, these patients had larger tumors and longer follow-up than the previously mentioned studies. Average tumor sizes in our study were $3.0 \mathrm{~cm}, 1.9 \mathrm{~cm}$ and $2.0 \mathrm{~cm}$, and recurrence rates were $0.81 \%, 0.67 \%$ and $0.72 \%$ for the mastectomy, NBCS and OBS groups, respectively. Our LRs were lower than those in the current literature, which might be due to the fact that all NBCS and OBS patients received postoperative boost RT. Additionally, our patients had smaller tumors and our mean follow-up duration was shorter than the above series.

Karanlik et al compared DFS and overall survival (OS) rates of EBC patients with mastectomy $(n=1209,66 \%)$, and NBCS $(n=632,34 \%) .{ }^{21}$ Five-year DFS and OS were $77 \%$ and $85 \%$ for patients with mastectomy, and $78 \%$ and $86 \%$ for patients with NBCS, respectively. The 5-year LR rate was $4 \%$ for the mastectomy group and $6 \%$ for NBCS. They concluded that age below 40 years and use of boost radiation were important determinants of LR in NBCS. Our comparison revealed DFS rates of 99.19\%, 99.33\%, and $99.27 \%$ respectively for mastectomy, NBCS, and OBS.

Oncoplastic resection of multifocal tumors has been shown to be oncologically safe. ${ }^{22}$ Positive margins have been reported at $2.7 \%-22 \%$ and have been associated with higher stage, positive nodes, positive lymphovascular invasion, use of neo-adjuvant CT, larger initial ' $\mathrm{T}$ ' stage, positive ER and younger age. ${ }^{23}$ Giacalone et al found that a significantly higher percentage of OBS patients had 5-10 mm free margins compared with patients with lumpectomies. ${ }^{24}$ Our data demonstrated no significant difference between tumor-free surgical margins of the NBCS and OBS groups.

The literature suggests that OBS decreases re-excision rates. ${ }^{25}$ Caruso et al evaluated the utility of intraoperative

Table 4. Adjuvant Therapy, Multicentricity, Multifocality and Recurrence

\begin{tabular}{|c|c|c|c|c|c|c|c|c|c|}
\hline & & \multirow[b]{2}{*}{$n$} & \multicolumn{3}{|c|}{ Group } & \multirow{2}{*}{$P$} & \multirow{2}{*}{$1-2 ; P$} & \multirow{2}{*}{$1-3 ; P$} & \multirow{2}{*}{$2-3 ; P$} \\
\hline & & & ${ }^{1} M$ & ${ }^{2} \mathrm{NBCS}$ & ${ }^{3} \mathrm{OBS}$ & & & & \\
\hline \multirow{2}{*}{ CT (\%) } & $(+)$ & 320 & $91(73.1)$ & $104(69.3)$ & $125(90.6)$ & \multirow{2}{*}{ b0.008** } & \multirow{2}{*}{${ }^{\mathrm{e}} 0.796$} & \multirow{2}{*}{${ }^{\mathrm{e}} 0.025^{*}$} & \multirow{2}{*}{${ }^{\mathrm{e}} 0.004^{* *}$} \\
\hline & $(-)$ & 92 & 33 (26.9) & $46(30.7)$ & $13(9.4)$ & & & & \\
\hline \multirow{2}{*}{ RT (\%) } & $(+)$ & 350 & $62(50.0)$ & $150(100)$ & $138(100)$ & \multirow{2}{*}{ b $0.001^{* *}$} & \multirow{2}{*}{ b $0.001^{* *}$} & \multirow{2}{*}{${ }^{\mathrm{e}} 0.001^{* *}$} & \multirow{2}{*}{ - } \\
\hline & $(-)$ & 62 & $62(50.0)$ & 0 & 0 & & & & \\
\hline \multirow{2}{*}{ HT (\%) } & $(+)$ & 292 & $86(69.2)$ & $118(78.7)$ & $88(64.1)$ & \multirow{2}{*}{ b0.153 } & \multirow{2}{*}{${ }^{\mathrm{e}} 0.319$} & \multirow{2}{*}{${ }^{\mathrm{e}} 0.698$} & \multirow{2}{*}{${ }^{\mathrm{e}} 0.085$} \\
\hline & $(-)$ & 120 & $38(30.8)$ & $32(21.3)$ & $50(35.9)$ & & & & \\
\hline \multirow{2}{*}{ MC/MF (\%) } & $(+)$ & 58 & $19(15.4)$ & $26(17.3)$ & $13(9.4)$ & \multirow{2}{*}{ bo.378 } & \multirow{2}{*}{${ }^{\mathrm{e}} 0.962$} & \multirow{2}{*}{${ }^{\mathrm{e}} 0.483$} & \multirow{2}{*}{${ }^{\mathrm{e}} 0.265$} \\
\hline & $(-)$ & 354 & $105(84.6)$ & $124(82.7)$ & $125(90.6)$ & & & & \\
\hline \multirow{2}{*}{ Recurrence (\%) } & $(+)$ & 3 & $1(0.8)$ & $1(0.7)$ & $1(0.7)$ & \multirow{2}{*}{${ }^{\mathrm{a}} 1.000$} & \multirow{2}{*}{${ }^{f} 1.000$} & \multirow{2}{*}{${ }^{\mathrm{f}} 1.000$} & \multirow{2}{*}{${ }^{f} 1.000$} \\
\hline & $(-)$ & 409 & $123(99.2)$ & 149 (99.3) & $137(99.3)$ & & & & \\
\hline $\begin{array}{l}\text { Time for recurrence } \\
\text { (month) }\end{array}$ & $\begin{array}{l}\text { Min-Max } \\
\text { (Median) } \\
\text { MeanSD }\end{array}$ & 412 & $\begin{array}{c}0-51(0) \\
48.21 \mathrm{SD} 2.33\end{array}$ & $\begin{array}{c}0-52(0) \\
49 S D 3.02\end{array}$ & $\begin{array}{c}0-50(0) \\
48.40 \text { SD1.62 }\end{array}$ & ${ }^{\circ} 0.963$ & - & - & ${ }^{8} 0.910$ \\
\hline
\end{tabular}

M, Mastectomy; NBCS, Non-oncoplastic breast conserving surgery; OBS, Oncoplastic breast surgery; CT, Chemotherapy; RT, Radiotherapy; HT, Endocrine therapy; MC/MF, Multicentric or multifocal; SD, Standard deviation.

${ }^{\text {a }}$ Fisher-Freeman-Halton test, ${ }^{\mathrm{b}}$ Pearson chi-square; ${ }^{\mathrm{c}}$ Kruskal-Wallis Test; e Yates continuity correction test; ${ }^{\mathrm{g}}$ Mann-Whitney test; ${ }^{*} P<0.05$; ${ }^{* *} P$ $<0.01$. 
Table 5. Disease-Free Survival Analysis

\begin{tabular}{lccccc}
\hline Group & N & R & DFS (\%) & DFS (months) & $\boldsymbol{P}$ \\
\hline Mastectomy & 124 & 1 & $99.19 \%$ & $81.635 \mathrm{SD} 1.047$ & \\
NBCS & 150 & 1 & $99.33 \%$ & $82.357 \mathrm{SD} 0.637$ & 0.937 \\
OBS & 138 & 1 & $99.27 \%$ & $82.081 \mathrm{SD} 0.414$ & \\
\hline
\end{tabular}

Kaplan-Meier Analysis: N, Number; R, Recurrence; DFS, Disease free survival; NBCS, Non-oncoplastic breast conserving surgery; OBS, Oncoplastic breast surgery

frozen section in patients undergoing therapeutic mammoplasty, and found that $8 / 52$ patients had positive frozen sections, with sensitivity of $83 \%$ and accuracy of $94 \%{ }^{26}$ In another study, the positive margin rate was significantly lower in the oncoplastic group (12\% vs. $21 \%, P<0.0001)$, and re-excision was more common in the NBCS group $(14.6 \%$ vs. $4 \%, P<0.0001)$; however, completion mastectomy was more common in the oncoplastic group $(6.5 \%$ vs. $3.79 \%, P<0.0001) .{ }^{27} \mathrm{We}$ recorded lower re-excision rates in the OBS (6.5\%) than the NBCS group (8\%). Our results for margin positivity and re-excision rates were slightly higher in BCS than OBS while the differences were not statistically significant.

Authors have reported low rates of completion mastectomy, although large long-term follow-up studies are lacking for OBS. The reported rates range from 5\% to $10 \%$, even when the tumors greater than $4 \mathrm{~cm}$ were included. ${ }^{22,28}$ We performed completion mastectomies in two cases with LR (1 OBS, 1 NBCS patient), and this rate is lower than that in the current literature.

The range of complication rates for OBS has been reported in the literature at $15 \%-30 \%{ }^{14,15}$ These complications include skin/flap necrosis, NAC necrosis, fat necrosis, seroma, hematoma, infection and wound dehiscence. Our complications such as wound dehiscence and extensive seroma formation were rare in all groups. Fat necrosis was slightly higher in the OBS group. Only one patient with OBS had type II diabetes mellitus, and developed partial NAC necrosis. These complication rates can be acceptable as they can be seen in all types of breast surgery.

DFS was $99.19 \%$ in the mastectomy, $99.33 \%$ in the NBCS and $99.27 \%$ in the OBS group.

A limitation of this study was that during the SLNB for axillary staging, we were unable to use the radioisotope method in support of blue dye in the axilla due to institutional restrictions. Also, the paucity of patients can be considered as another limitation.

In conclusion, mastectomy, NBCS, and OBS are equally acceptable procedures in EBC in terms of oncological safety. Re-excision rates are lower in OBS than NBCS, and LR are similar in all groups during follow-up. The results demonstrated that DFS was not different between the groups. Incorporation of OBS into EBC treatment appears oncologically and surgically acceptable.

\section{Authors' Contribution}

Design of study: JS; literature search; JS, OO, FA; data collection: JS, ETB, ABC, TO, AG; verification of data and statistical analysis: JS, TE, IAO, HB; final supervision: $\mathrm{OA}, \mathrm{GB}, \mathrm{OE}$; manuscript writing: JS.

\section{Conflict of Interest Disclosures}

The authors declare that they have no conflict of interest.

\section{Ethical Statement}

This study was conducted in accordance with the amended Declaration of Helsinki. An informed consent was obtained from all patients upon admission according to the Ethical Board requirements.

\section{Funding}

This research did not receive any specific grant from funding agencies in the public, commercial, or not-for-profit sectors.

\section{Acknowledgements}

The authors acknowledge that the final manuscript has been prepared and submitted with their approval. The authors thank all hospital staff for their support.

\section{References}

1. Fisher B, Jeong JH, Anderson S, Bryant J, Fisher ER, Wolmark N. Twenty-five-year follow-up of a randomized trial comparing radical mastectomy, total mastectomy, and total mastectomy followed by irradiation. N Engl J Med. 2002;347(8):567-75. doi: 10.1056/NEJMoa020128.

2. Fisher B, Anderson S, Bryant J, Margolese RG, Deutsch M, Fisher ER, et al. Twenty-year follow-up of a randomized trial comparing total mastectomy, lumpectomy, and lumpectomy plus irradiation for the treatment of invasive breast cancer. N Engl J Med. 2002;347(16):1233-41. doi: 10.1056/NEJMoa022152.

3. Morrow M. Limiting breast surgery to the proper minimum. Breast. 2005;14(6):523-6. doi: 10.1016/j.breast.2005.08.008.

4. Rainsbury R. Surgery insight: oncoplastic breast conserving reconstruction-indications, benefits, choices and outcomes. Nat Clin Pract Oncol. 2007;4(11):657-64. doi: 10.1038/ ncponc0957.

5. Piper M, Peled AW, Sbitany H. Oncoplastic breast surgery: current strategies. Gland Surg. 2015;4(2):154-63. doi: 10.3978/j.issn.2227-684X.2015.03.01.

6. Clough KB, Ihrai T, Oden S, Kaufman G, Massey E, Nos C. Oncoplastic surgery for breast cancer based on tumour location and a quadrant-per-quadrant atlas. Br J Surg. 2012;99(10):1389-95. doi: 10.1002/bjs.8877.

7. Veronesi U, Cascinelli N, Mariani L, Greco M, Saccozzi $\mathrm{R}$, Luini A, et al. Twenty-year followup of a randomized study comparing breast-conserving surgery with radical mastectomy for early breast cancer. $\mathrm{N}$ Engl J Med. 2002;347(16):1227-32. doi: 10.1056/NEJMoa020989.

8. McCulley SJ, Durani P, Macmillan RD. Therapeutic mammaplasty for centrally located breast tumors. Plast Reconstr Surg. 2006;117(2):366-73. doi: 10.1097/01. prs.0000200874.31320.c2.

9. Losken A, Pinell-White X, Hart AM, Freitas AM, Carlson GW, Styblo TM. The oncoplastic reduction approach to breast conservation therapy: benefits for margin control. Aesthet Surg J. 2014;34(8):1185-91. doi: $10.1177 / 1090820 X 14545618$. 
10. Chan SWW, Cheung PSY, Lam SH. Cosmetic outcome and percentage of breast volume excision in oncoplastic breast conserving surgery. World J Surg. 2010;34(7):1447-52. doi: 10.1007/s00268-009-0278-x.

11. Chang EI, Peled AW, Foster RD, Lin C, Zeidler KR, Ewing $\mathrm{CA}$, et al. Evaluating the feasibility of extended partial mastectomy and immediate reduction mammoplasty reconstruction as an alternative to mastectomy. Ann Surg. 2012;255(6):1151-7. doi: 10.1097/SLA.0b013e31824f9769.

12. Clough KB, Lewis JS, Couturaud B, Fitoussi A, Nos C, Falcou MC. Oncoplastic techniques allow extensive resections for breast-conserving therapy of breast carcinomas. Ann Surg. 2003;237(1):26-34. doi: 10.1097/00000658-20030100000005.

13. Berry MG, Fitoussi AD, Curnier A, Couturaud B, Salmon RJ. Oncoplastic breast surgery: a review and systematic approach. J Plast Reconstr Aesthet Surg. 2010;63(8):123343. doi: 10.1016/j.bjps.2009.05.006.

14. Iwuchukwu OC, Harvey JR, Dordea M, Critchley AC, Drew PJ. The role of oncoplastic therapeutic mammoplasty in breast cancer surgery--a review. Surg Oncol. 2012;21(2):13341. doi: 10.1016/j.suronc.2011.01.002.

15. Rietjens M, Urban CA, Rey PC, Mazzarol G, Maisonneuve $\mathrm{P}$, Garusi C, et al. Long-term oncological results of breast conservative treatment with oncoplastic surgery. Breast. 2007;16(4):387-95. doi: 10.1016/j.breast.2007.01.008.

16. Barnea $\mathrm{Y}$, Inbal A, Barsuk D, Menes T, Zaretski A, Leshem $D$, et al. Oncoplastic reduction using the vertical scar superior-medial pedicle pattern technique for immediate partial breast reconstruction. Can J Surg. 2014;57(4):E13440. doi: $10.1503 /$ cjs.031213.

17. Anderson BO, Masetti R, Silverstein MJ. Oncoplastic approaches to partial mastectomy: An overview of volumedisplacement techniques. Lancet Oncol. 2005;6(3):145-57. doi: 10.1016/S1470-2045(05)01765-1.

18. Losken A, Styblo TM, Carlson GW, Jones GE, Amerson BJ. Management algorithm and outcome evaluation of partial mastectomy defects treated using reduction or mastopexy techniques. Ann Plast Surg. 2007;59(3):235-42. doi:10.1097/SAP.0b013e31802ec6d1.

19. Caruso F, Catanuto G, De Meo L, Ferrara M, Gallodoro A, Petrolito E, et al. Outcomes of bilateral mammoplasty for early stage breast cancer. Eur J Surg Oncol. 2008;34(10):1143-
7. doi: 10.1016/j.ejso.2007.09.012.

20. Bogusevicius A, Cepuliene D, Sepetauskiene E. The integrated evaluation of the results of oncoplastic surgery for locally advanced breast cancer. Breast J. 2014;20(1):5360. doi: 10.1111/tbj.12222.

21. Karanlık H, Özmen V, Asoğlu O, Igci A, Kecer M, Tuzlalı $S$, et al. Meme kanseri cerrahi tedavisinin uzun dönem sonuçlari (Long term results of surgical treatment for breast cancer). Meme Sagligi Dergisi (J Breast Health). 2006;2(2):89-95.

22. Patani N, Carpenter R. Oncological and aesthetic considerations of conservational surgery for multifocal/ multicentric breast cancer. Breast J. 2010;16(3):222-32. doi: 10.1111/j.1524-4741.2010.00917.x.

23. Chang E, Johnson N, Webber B, Booth J, Rahhal D, Gannett $\mathrm{D}$, et al. Bilateral reduction mammoplasty in combination with lumpectomy for treatment of breast cancer in patients with macromastia. Am J Surg. 2004;187(5):647-50. doi: 10.1016/j.amjsurg.2004.01.002.

24. Giacalone PL, Roger P, Dubon O, El Gareh N, Rihaoui S, Taourel P, et al. Comparative study of the accuracy of breast resection in oncoplastic surgery and quadrantectomy in breast cancer. Ann Surg Oncol. 2007;14(2):605-14. doi: 10.1245/s10434-006-9098-5.

25. Chakravorty A, Shrestha AK, Sanmugalingam N, Rapisarda F, Roche N, Della Rovere GQ, et al. How safe is oncoplastic breast conservation? Comparative analysis with standard breast conserving surgery. Eur J Surg Oncol. 2012;38(5):3958. doi: 10.1016/j.ejso.2012.02.186.

26. Caruso F, Ferrara M, Castiglione G, Cannata I, Marziani A, Polino C, et al. Therapeutic mammaplasties: full local control of breast cancer in one surgical stage with frozen section. Eur J Surg Oncol. 2011;37(10):871-5. doi: 10.1016/j. ejso.2011.07.002.

27. Losken A, Dugal CS, Styblo TM, Carlson GW. A metaanalysis comparing breast conservation therapy alone to the oncoplastic technique. Ann Plast Surg. 2014;72(2):1459. doi: 10.1097/SAP.0b013e3182605598.

28. Bong J, Parker J, Clapper R, Dooley W. Clinical series of oncoplastic mastopexy to optimize cosmesis of largevolume resections for breast conservation. Ann Surg Oncol. 2010;17(12):3247-51. doi: 10.1245/s10434-010-1140-y. 